\title{
Vancomycin Resistant Staphylococcus aureus Reported from Tertiary Care Hospital in Nepal
}

\author{
Urmila Lama ${ }^{1}$, Dharmendra Shah ${ }^{2}$, Upendra Thapa Shrestha ${ }^{3}$ \\ ${ }^{1}$ Department of Microbiology, Kantipur College of Medical Science, Sitapaila, Kathmandu \\ ${ }^{2}$ Manmohan Memorial Medical College and Teaching Hospital, Swoyambhu, Kathmandu \\ ${ }^{3}$ Central Department of Microbiology, Tribhuvan University, Kirtipur, Kathmandu
}

*Corresponding author: Upendra Thapa Shrestha, Central Department of Microbiology, Tribhuvan University, Kirtipur Email: upendrats@microbiotu.edu.np

\begin{abstract}
Objectives: The study was conducted to assess the rate of Methicillin-resistant Staphylococcus aureus (MRSA) among patients and healthcare personnel at Manmohan Memorial College and Teaching Hospital, Kathmandu, Nepal and to evaluate the minimum inhibitory concentration of Vancomycin to MRSA isolates.
\end{abstract}

Methods: A total of 1433 different clinical specimens from patients and 33 nasal swabs from healthcare personnel were subjected to bacteriological investigation following standard protocol. S. aureus were isolated and identified by using standard Microbiological tools. Those isolates were subjected to Antimicrobial susceptibility testingusing modified Kirby-Bauer's disc diffusion method following CLSI guidelines.

Results: The rate of S. aureus carriage was found to be $65(18.9 \%)$ in the samples from clinical patients and $24(72.7 \%)$ in the samples from healthcare personnel. The rate of MRSA was found to be $57(85.1 \%)$ in patients and $24(100 \%)$ in healthcare personnel. The high distribution of MRSA was found in female of age group 21-30 years (patients: 10.4\%; healthcare personnel: 70.8\%). Amikacin was found to be most effective antimicrobial. All S. aureus isolates were found to be multidrug resistant (100\%).On performing D-test, 10 (17.5\%) and 22 (38.6\%) of MRSA from clinical specimens showed inducible and constitutive Clindamycin resistance respectively. Whereas, 11 (45.8\%) and 4 (16.7\%) of MRSA from nasal swabs were found to be inducible and constitutive Clindamycin resistance respectively. Upon performing minimum inhibitory concentration (MIC) test for clinical isolates, 3.5\% (2) of MRSA were found to be Vancomycin resistant (VRSA), 54.4\% (31) were Vancomycin intermediate (VISA) and 42.1\% (24) were found to be Vancomycin sensitive (VSSA). All of the nasal swab MRSA isolates were found sensitive to Vancomycin. Congo red agar method was done for biofilm production. For clinical isolates, 32 (47.8\%) were found to be strong, 6 (8.9\%) moderate and 29 (43.3\%) were non biofilm producer. For nasal swab isolates, $66.7 \%$ (16) and $33.3 \%$ (8) were found as strong and non-biofilm producer respectively.

Conclusion: This study reported the case of VRSA which hasn't been reported in Nepal. Though present study showed that Vancomycin remains the main choice of treatment of MRSA infection. Therefore, to preserve its value, use of vancomycin should be limited only to those cases where there are clearly needed.

Key words: S. aureus, MRSA, D-test, Inducible Clindamycin resistance,VRSA

\section{INTRODUCTION}

Staphyolococcus aureus is one of the common human pathogens capable of causing a wide range of infections. A great deal of virulence from the organism occurs through cross infection by patient to patient in hospitals and other institutional settings. In contrast, healthy individuals have a small risk of invasive infection caused by $S$. aureus, but they can 
be carriers of the organism (Foster 2004). Infection due to Methicillin-Resistant S. aureus (MRSA) are an increasing problem worldwide in community as well as hospital environment (Boyce et al. 2005; Skoy et al. 2006). The incidence of community-acquired and hospital-acquired $S$. aureus infections has been rising with increasing emergence of drug-resistant strains called Methicillin resistant S. aureus (MRSA) (Steinberg et al. 1996).

The resistance of $S$. aureus to Methicillin is caused by the mecA gene which codes the low affinity 78-Kda penicillin-binding protein (PBP2a). Betalactam antibiotic normally binds to PBPs in the cell wall, resulting in the disruption of synthesis of the peptidoglycan layer and death of bacterium. Since the beta-lactam antibiotics cannot bind to low affinity PBP2a, synthesis of peptidoglycan layer and cell wall are able to continue (Duerenberg 2007). MRSA infections often require systematic antibiotic therapy. The spread of MRSA can also be potentially minimized by prevention of the risk factors such as previous antibiotic use, contact with the healthcare workers or nursing home resident, daycare attendance, hospitalization, admission to an intensive care unit, intravenous drug use, invasive indwelling devices, haemodialysis or peritoneal dialysis, immunosuppression, chronic illness, and previous isolation of MRSA (Cohen 2007).

Following the spread of MRSA, glycopeptides (usually Vancomycin and more recently Teicoplanin) have become the mainstay of treatment for MRSA infections (CDC 2013). Vancomycin is the choice of drug for MRSA isolates. Patients unable to tolerate vancomycin have been treated with fluoroquinolones, TrimethoprimSulfomethoxazole, Clindamycin or Minocycline (Shah 2008). As Vancomycin is commonly used for the treatment of MRSA infections, which has resulted into development of Vancomycin-Intermediate S. aureus (VISA) and Vancomycin-Resistant S. aureus (VRSA).

Clindamycin, a lincosamide drug, has been used to treat serious infections caused by susceptible $S$. aureus in children for more than 30 years (Woods 2009). It is also an alternative choice in case of Penicillin allergic patients. Clindamycin is recommended in some European countries for suppression of panton-valentine leukocidin (PVL) toxin, along with Linezolid and Rifampin (Adaleti et al. 2010). In vitro, S. aureus isolates with constitutive resistant are resistant to Erythromycin and Clindamycin while isolates with inducible resistant are resistant to Erythromycin but appear susceptible to Clindamycin (Steward et al. 2005). Inducible MLSB (Macrolide, Lincosamide and Streptogramin B) resistant can be detected by discapproximation test (Dtest) by placing Erythromycin and Clindamycin discs in adjacent positions (Fiebelkorn et al. 2003).

Biofilms are communities of microorganisms embedded in extracellular polymeric substances (EPS) matrix. Bacteria in biofilms demonstrate distinct features from their freeliving planktonic counterparts, such as different physiology and high resistance to immune system and antibiotics that render biofilm a source of chronic and persistent infections. Extracellular polymeric matrix plays various roles in structure and function of different biofilm communities. Adhesion to the surface provides considerable advantages such as protection against antimicrobial agent, acquisition of new genetic traits and the nutrients availability and metabolic co-operability. Bacterial biofilms cause chronic infections because they show increased tolerance to antibiotics and disinfectant chemicals as well as resisting phagocytosis and other components of the body's defense system (Donlan and Costerton 2002).

The study was thus done to determine the rate of Methicillin resistant, emergence of Vancomycin resistant and inducible Clindamycin resistant $S$. aureus among biofilm producing and non-producing isolates of S. aureus.

\section{MATERIALS AND METHODS}

Study design: A hospital based cross sectional descriptive study was conducted.

Study period, site and population: The study was conducted at Manmohan Memorial Medical College and Teaching Hospital, Swoyambhu, Kathmandu, Nepal in collaboration with Kantipur College of Medical Science, Sitapaila, Kathmandu, Nepal from April 2014 to October 2014. All the clinical specimens obtained from individuals of all ages and sexes visiting hospital during the study period were included in the study.

Sample size: A total of 1344 clinical specimens including blood, urine, sputum, vaginal swab, eye swab, ear swab, throat swab, wound swab, clavical swab, body fluids like pus, synovial fluid, pleural fluid, asiatic fluid, peritoneal fluid and catheter swabs and urethral discharge; were processed in the study. For the study of hospital acquired MRSA, a total of 33 nasal swabs were collected from the hospital personnel. 


\section{Laboratory diagnosis}

Sample collection: Sterilized sample collection container was used for the collection of all clinical specimens. Blood was collected with sterile syringe and then poured in leak proof, dry and sterilized container.

Sample processing and bacterial identification: All the clinical samples were inoculated onto blood agar, chocolate agar and mac-conkey agar plates. Blood samples were inoculated into brain heart infusion broth and incubated at $37^{\circ} \mathrm{C}$ for 7 days and then further inoculated into agar media. All the culture plates were then incubated at $37^{\circ} \mathrm{C}$ for overnight. The plates showing growth of bacterial were processed for identification of $S$. aureus using standard microbiological procedures by inoculating the organism on mannitol salt agar and performing specific biochemical tests catalase test, coagulase test and oxidative-fermentative test.

Antimicrobial susceptibility testing and screening of multi drug resistant (MDR) S. aureus: All the identified isolates of $S$. aureus were undertaken in-vitro antibiotic susceptibility test by using modified Kirby-Bauer's disc diffusion method (CLSI 2013). The antibiotics used were Cefoxitin ( $5 \mathrm{mcg})$, Ciprofloxacin $(5 \mathrm{mcg})$, Cefixime $(5 \mathrm{mcg})$, Tetracycline (30mcg), Amikacin (30mcg), Azithromycin (30mcg), Vancomycin (30mcg), Cloxacillin (5mcg), Cefotaxime (30mcg), Clindamycin (10mcg), Ceftriaxone (30mcg), Erythromycin (15mcg), Gentamicin (10mcg), Penicillin (10mcg), Co-trimoxazole (25mcg), Mupirocin (5mcg), and Chloramphenicol (50mcg). The organism resistant to three or more antibiotics of different classes were classified as MDR isolates (Magiorakos et al. 2012). Intrinsic resistance to any of the employed antibiotics was not counted.

Screening of methicillin resistant S. aureus: Screening for Methicillin resistantS. aureus was carried out by Cefoxitin disc diffusion method and interpreted according to CLSI (2013) guidelines. The growth of $S$. aureus with zone of inhibition around Cefoxin disc $(\mathrm{ZOI}) \geq 22 \mathrm{~mm}$ were identified as Methicillin sensitive $S$. aureus and that of $\mathrm{ZOI} \leq 21$ were identified as Methicillin resistant $S$. aureus.
Detection of Inducible clindamycin resistance (ICR): In this assay, two discs namely Erythromycin and Clindamycin were placed $18 \mathrm{~mm}$ away edge-to-edge on Muller Hinton agar plates that were previously inoculated with 0.5 McFarland bacterial suspensions. Plates were observed after 18 hours of incubation at $35 \pm 2{ }^{\circ} \mathrm{C}$. Flattening of the zone of inhibition adjacent to the Erythromycin disc (referred to as D-zone) or hazy growth within the zone of inhibition around Clindamycin (even if no D- zone is apparent) is regarded as positive test, i.e. Inducible Clindamycin resistance (CLSI 2013).

Determination of minimum inhibitory concentration of vancomycin: Minimum inhibitory concentration (MIC) technique was performed to determine the Vancomycin intermediate and resistant strains of $S$. aureus isolates MIC to Vancomycin in isolated MRSA was done by agar dilution method following CLSI guidelines (CLSI 2013). Different concentrations ranging from $0.06-32 \mu \mathrm{g} / \mathrm{ml}$ of Vancomycin incorporated plates was prepared. Positive growth controls were kept for each isolates and S. aureus (ATCC 25923) of known MIC was also included in each test as control for antibiotic potency.

Biofilm production: Biofilm detection was carried out by Congo Red Agar method (CRA): CRA medium was prepared with brain heart infusion broth $37 \mathrm{~g} / \mathrm{L}$, sucrose $50 \mathrm{~g} / \mathrm{L}$, agar $10 \mathrm{~g} / \mathrm{L}$ and Congo Red indicator 8 g/L. Congo Red stain was prepared as a concentrated aqueous solution and autoclaved separately from the other medium constituents. Then it was added to the autoclaved brain heart infusion agar with sucrose. CRA plates was inoculated with test organisms and incubate at $37^{\circ} \mathrm{C}$ for overnight aerobically. Black colonies were considered as biofilm producing isolates (Freeman et al. 1989).

\section{RESULTS}

Out of total 1433 clinical specimens, S. aureus was isolatesd from 67 specimens $(4.7 \%)$, among them 57 (85.1\%) were found to be MRSA. Whereas from 33 nasal swab specimens, 24 (72.7\%) S. aureus were isolated and all of them were found to be MRSA (100\%). All of the $S$. aureus isolates from clinical as well as nasal swab specimens were multi-drug resistant (MDR) (Figure 1). 


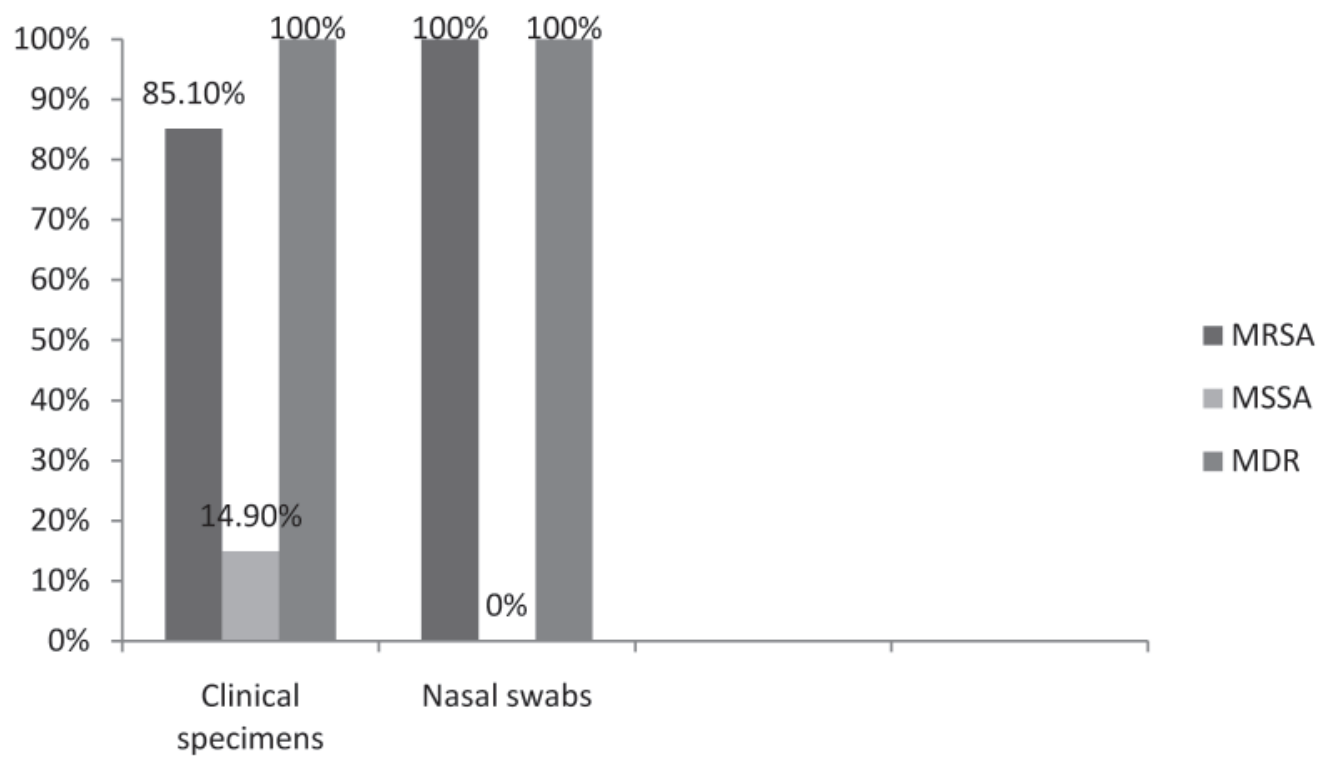

Figure 1: Distribution of MRSA, MSSA and MDR S. aureus

Among total of 67 S. aureus isolates, 27 (40.3\%) from male and 30 (44.8\%) from female. High rate of MRSA 12
(17.9\%) was obtained from age group 21-30 year (Table $1)$.

Table 1: Age and sex wise distribution of MRSA from clinical specimens

\begin{tabular}{lcccccccc}
$\begin{array}{l}\text { Age } \\
\text { group } \\
\text { (year) }\end{array}$ & $\begin{array}{c}\text { Total No. of } \\
\text { samples }\end{array}$ & \multicolumn{3}{c}{ Male Number (\%) } & \multicolumn{3}{c}{ Female Number (\%) } & Total no. of \\
\cline { 3 - 7 } & Samples & S. aureus & MRSA & Samples & S. aureus & MRSA & MRSA (\%) \\
$11-20$ & 109 & 56 & $3(4.5)$ & $2(2.9)$ & 53 & $1(1.5)$ & $1(1.5)$ & $3(4.5)$ \\
$21-30$ & 204 & 99 & $7(10.4)$ & $4(5.9)$ & 105 & $2(2.9)$ & $2(2.9)$ & $6(9)$ \\
$31-40$ & 382 & 121 & $6(9)$ & $5(7.5)$ & 261 & $7(10.4)$ & $7(10.4)$ & $12(17.9)$ \\
$41-50$ & 202 & 86 & $3(4.5)$ & $2(2.9)$ & 116 & $4(5.9)$ & $4(5.9)$ & $6(9)$ \\
$51-60$ & 124 & 65 & $6(9)$ & $6(9)$ & 59 & $4(5.9)$ & $4(5.9)$ & $10(14.9)$ \\
$61-70$ & 128 & 40 & $1(1.5)$ & $1(1.5)$ & 88 & $6(9)$ & $5(7.5)$ & $6(9)$ \\
$71-80$ & 108 & 55 & $3(4.5)$ & $3(4.5)$ & 53 & $5(7.5)$ & $3(4.5)$ & $6(9)$ \\
$>80$ & 87 & 37 & $2(2.9)$ & $2(2.9)$ & 50 & $3(4.5)$ & $2(2.9)$ & $4(5.9)$ \\
\hline Total & 89 & 51 & $2(2.9)$ & $2(2.9)$ & 38 & $2(2.9)$ & $2(2.9)$ & $4(5.9)$ \\
\hline
\end{tabular}

Among 24 nasal swab MRSA isolates, 2 (8.3\%) were from male and $22(91.7 \%)$ were from female and High rate of MRSA was 19 (79.2\%) obtained from age group 21-30 year (Table 2 ).

Table 2: Age and sex wise distribution of MRSA from nasal swabs

\begin{tabular}{|c|c|c|c|c|c|c|c|c|}
\hline \multirow{2}{*}{$\begin{array}{l}\text { Age group } \\
\text { (year) }\end{array}$} & \multirow{2}{*}{$\begin{array}{l}\text { Total no. of } \\
\text { samples }\end{array}$} & \multicolumn{3}{|c|}{ Male Number (\%) } & \multicolumn{3}{|c|}{ Female Number (\%) } & \multirow{2}{*}{$\begin{array}{c}\text { Total no } \\
\text { of MRSA } \\
\text { (\%) }\end{array}$} \\
\hline & & Samples & S. aureus & MRSA & Samples & S. aureus & MRSA & \\
\hline$<10$ & - & - & - & - & - & - & - & - \\
\hline $11-20$ & 4 & 1 & - & - & 3 & $2(8.3)$ & $2(8.3)$ & $2(8.3)$ \\
\hline $21-30$ & 22 & 3 & $2(8.3)$ & $2(8.3)$ & 21 & $\begin{array}{c}17 \\
(70.8)\end{array}$ & $\begin{array}{c}17 \\
(70.8)\end{array}$ & $\begin{array}{c}19 \\
(79.2)\end{array}$ \\
\hline $31-40$ & 7 & - & - & - & 4 & $3(12.5)$ & $3(12.5)$ & $3(12.5)$ \\
\hline Total & 33 & 4 & $\begin{array}{c}2 \\
(8.3)\end{array}$ & $\begin{array}{c}2 \\
(8.3)\end{array}$ & 29 & $\begin{array}{c}22 \\
(91.7)\end{array}$ & $\begin{array}{c}22 \\
(91.7)\end{array}$ & $\begin{array}{c}24 \\
(100)\end{array}$ \\
\hline
\end{tabular}


MRSA from clinical specimens were $100 \%$ resistant to Penicillin G, Co-trimoxazole and Cefixime, followed by Cloxacillin (94.7\%), Ceftriaxone (93\%), Vancomycin $(92.2 \%)$, and Cefotaxime (84.2\%). Whereas MMSA isolates were $100 \%$ resistant to Cefixime, followed by Cefotaxime (90\%), Ceftriaxone (90\%), Penicillin G
(80\%) and Co-trimoxazole (80\%). For nasal swab MRSA isolates, $100 \%$ showed resistance towards Cefixime, Cefotaxime, Penicillin G and Co-trimoxazole, followed by Cloxacillin (75\%), Clindamycin (54.2\%), and Tetracycline (50\%). Whereas no MMSA isolates were obtained (Table 3).

Table 3: Antimicrobial resistance profile of MRSA and MSSA

\begin{tabular}{lcccc}
\hline \multirow{2}{*}{ Antibiotics } & \multicolumn{2}{c}{ Clinical specimens } & \multicolumn{2}{c}{ Nasal swab specimens } \\
\cline { 2 - 5 } & $\begin{array}{c}\text { Antibiotic profile of } \\
\text { MRSA ( } \mathrm{n}=57)\end{array}$ & $\begin{array}{c}\text { Antibiotic profile of } \\
\text { MSSA }(\mathrm{n}=10)\end{array}$ & $\begin{array}{c}\text { Antibiotic profile of } \\
\text { MRSA }(\mathrm{n}=24)\end{array}$ & $\begin{array}{c}\text { profile of MSSA } \\
\text { Antibiotic }(\mathrm{n}=0)\end{array}$ \\
\hline Cefoxitin & $57(100 \%)$ & - & $24(100 \%)$ & - \\
Ciprofloxacin & $15(26.3 \%)$ & $3(30 \%)$ & $8(33.3 \%)$ & - \\
Cefixime & $57(100 \%)$ & $10(100 \%)$ & $24(100 \%)$ & - \\
Tetracyclin & $32(56.1 \%)$ & - & $12(50 \%)$ & - \\
Amikacin & $8(14 \%)$ & - & $4(16.7 \%)$ & - \\
Azithromycin & $25(43.9 \%)$ & $1(10 \%)$ & $6(25 \%)$ & - \\
Vancomycin & $53(92.9 \%)$ & $4(40 \%)$ & $8(33.3 \%)$ & - \\
Cloxacillin & $54(94.7 \%)$ & $1(10 \%)$ & $18(75 \%)$ & - \\
Cefotaxime & $48(84.2 \%)$ & $9(90 \%)$ & $24(100 \%)$ & - \\
Clindamycin & $42(73.7 \%)$ & $4(40 \%)$ & $13(54.2 \%)$ & - \\
Ceftriaxone & $53(93 \%)$ & $9(90 \%)$ & $24(100 \%)$ & - \\
Erythromycin & $32(56.1 \%)$ & - & $8(33.3 \%)$ & - \\
Gentamicin & $13(22.8 \%)$ & - & $4(16.7 \%)$ & - \\
Penicillin G & $57(100 \%)$ & $8(80 \%)$ & $24(100 \%)$ & - \\
Co-trimoxazole & $57(100 \%)$ & $8(80 \%)$ & $24(100 \%)$ & - \\
Mupirocin & $43(75.4 \%)$ & $3(30 \%)$ & $18(75 \%)$ & - \\
Cloramphenicol & $11(19.3 \%)$ & - & $3(12.5 \%)$ & \\
\hline
\end{tabular}

Upon performing D-test, 10 (17.5\%) and 22 (38.6\%) of MRSA from clinical specimens showed inducible and constitutive Clindamycin resistance respectively.
Whereas, $11(45.8 \%)$ and 4 (16.7\%) of MRSA from nasal swabs were found to be inducible and constitutive Clindamycin resistance respectively (Table 4).

Table 4: D-test of MRSA isolates

\begin{tabular}{lccccc}
\hline \multirow{2}{*}{ Phenotype } & Erythromycin & Clindamycin & D-test & \multicolumn{2}{c}{ MRSA (\%) } \\
\cline { 5 - 6 } & & & Clinical isolates & Nasal swab isolates \\
\hline iMLSB & Resistance & Sensitive & Positive & $10(17.5)$ & $11(45.8)$ \\
CMLSB & Resistance & Resistance & Negative & $22(38.6)$ & $4(16.7 \%)$ \\
\hline
\end{tabular}

From clinical specimens, $47.8 \%$ (32), $8.9 \%$ (6) and $43.3 \%$ (29) of S. aureus isolates were found to be strong, moderate and non-biofilm producer respectively.
Among nasal swab specimens, 66.7\% (16) and 33.3\% (8) of $S$. aureus isolates were found to be strong and nonbiofilm producer respectively (Table 5).

Table 5: Biofilm production by $S$. aureus

\begin{tabular}{lccc}
\hline Serial no. & Strong producer & Moderate producer & Non producer \\
\hline $\begin{array}{l}\text { 1.clinical specimen } \\
(\mathrm{n}=67)\end{array}$ & $32(47.8 \%)$ & $6(8.9 \%)$ & $29(43.3 \%)$ \\
2.Nasal swab $(\mathrm{n}=24)$ & $16(66.7)$ & - & $8(33.3 \%)$ \\
\hline
\end{tabular}


On performing MIC of MRSA from clinical specimens, $42.1 \%$ (24) of isolates were reported as VSSA (showed MIC value of $2 \mu \mathrm{g} / \mathrm{ml}$ ), $54.4 \%$ (31) as VISA (showed MIC value of $4-8 \mu \mathrm{g} / \mathrm{ml}$ ) and $3.5 \%$ (2) of MRSA isolates were reported as VRSA (showed MIC value of $\geq 16 \mu \mathrm{g} / \mathrm{ml}$ ) (Figure 2). Whereas all MRSA isolates from nasal swabs were found to be Vancomycin sensitive (VSSA).

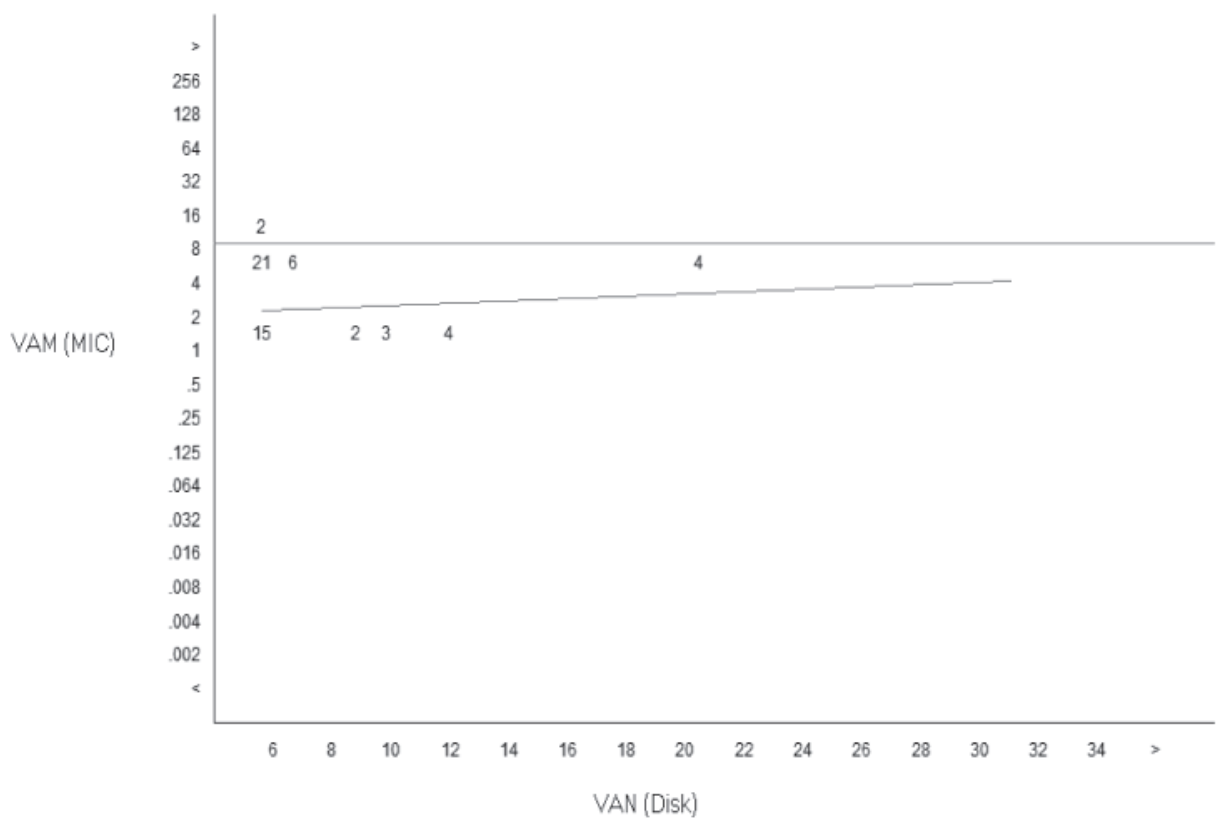

Figure 2: Scatter plot analysis of MIC of vancomycin for MRSA isolates from clinical specimens (WHONET 5.6)

\section{DISCUSSION}

S. aureus has remained a versatile and potent pathogen in humans, since it is one of the most common causes of nosocomial and community acquired infections (Rajbhandari et al. 2003). S. aureus is a major cause of infectious morbidity and mortality around the world, causing a wide variety of clinical manifestations ranging from localized infection to toxin mediated diseases and invasive blood stream infections(Vandecasteele et al. 2008).

In this study, the rate of MRSA isolation was found to be $85.1 \%$ from clinical specimens. This result is higher than the many other studies conducted by Kumari et al. (26.14\%), Shakya et al. (12.5\%) and Tiwari et al. (69.1\%) (Tiwari et al. 2006; Kumari et al. 2008; Shakya et al. 2010). All of the $S$. aureus isolates from nasal swab specimens were found to be MRSA i.e. 100\%. Nasal carriage rate of MRSA among health care workers in hospital setting ranges from 6-17.8\% (Cesur and Cokca 2004; Pant and Rai 2007). Nasal carriage rate of $43.8 \%$ has been reported among the healthcare personnel of a Medical College Teaching Hospital in Kathmandu (Pant and Rai 2007). The nasal carriage rate of S. aureus in this study i.e. $72.7 \%$ was found to be greater than the study conducted by Shakya et al. i.e. 12.5\% (Shakya et al. 2010). The result is also in agreement with the study by Gonsu et al. (Gonsu et al. 2013).

Regarding the sex wise distribution of MRSA clinical specimens, the study showed high incidence of MRSA from female patients $(44.8 \%)$ than males $(40.3 \%)$. The present study showed the opposite variation with the study conducted by Boucher and Corey (Boucher and Corey 2008) showing males (64.4\%) were more predisposed than females (35.6\%). The highest distribution of MRSA was found within the age group of $21-30$ years $(17.9 \%)$ and the lowest in the age group below 10 years $(4.5 \%)$. However, the study conducted by Arch et al. (Arch et al. 2006) and Lucet et al. (Lucet et al. 2003) showed high rate of MRSA colonization among the population with age group 60 years and above.

The nasal carriage rate of MRSA reported in present study was found to be higher $(72.7 \%$ ) than the previous studies conducted in Nepal by Shakya et al. and Rijal et al. (Rijal et al. 2008; Shakya et al. 2010).

Penicillin was found resistant to all of MRSA isolates 
i.e. $100 \%$. This result is higher than that of Shrestha et al. who reported $91.94 \%$ (Shrestha et al. 2009). In present study, clinical MRSA isolates showed rate of resistance to antibiotics Co-trimoxazole (100\%), followed by Cloxacillin (94.7\%), Ceftriazone (93\%), Vancomycin (92.9\%), Cefotaxime (84.2\%), Mupirocin (75.4\%), Clindamycin (73.7\%), Tetrcyclin (56.1\%) and Erythromycin (56.1\%). Rijal et al. reported the rate of resistance to Cloxacillin (68.8\%), followed by Tetracycline (15.6\%) and Erythromycin (9.4\%) (Rijal et al. 2008). Resistance to Erythromycin is seen to be greater than the finding disseminated by study conducted by Mishra i.e. 14.29\% (Mishra 2008)and lower than the finding disseminated by Tiwari et al. i.e. $68.7 \%$ (Tiwari et al. 2006).

All isolates were found to be multi drug resistant (MDR) in this study. The rate of MDR-MRSA (100\%) is higher than that of the result reported in the studies conducted by Tiwari et al. i.e. $40.1 \%$ and Pandey et al. i.e. $75.86 \%$. Though this study is in accordance with the previous studies from Nepal and other countries showing high percentage of MDR among MRSA; >65\% by Kumari et al., $93 \%$ by Rahimi et al. and $63 \%$ by Salah et al. (Kumari et al. 2008; Salah et al. 2012; Rahimi et al. 2013).

In this study, $17.5 \%$ and $38.6 \%$ isolates were found to be inducible and constitutive Clindamycin resistance respectively. Among nasal swab specimens taken from hospital staffs, $45.5 \%$ and $16.7 \%$ were found to be inducible and constitutive Clindamycin resistance respectively. In the study conducted by Ujwol et al. (Bhomi et al. 2016), D-test positive isolates were found to be $18.03 \%$ and study also reported constitutive resistance in $36.06 \%$ of isolates.

Upon performing MIC, 3.5\% (Skoy et al. 2006)) of clinical MRSA isolates were reported as VRSA, 54.4\% (31) as VISA and 24.1\% (Vandecasteele et al. 2008) as VSSA. Assadullah et al. (Assadullah et al. 2003); Sharma and Vishwanath (Sharma and Vishwanath 2012) reported $18.3 \%$ and $11.54 \%$ VISA among MRSA respectively. In the study carried out by Venubabu et al. (Venubabu et al. 2011), who reported 1.9\% VRSA from India. Likewise Tiwari and Sen (Tiwari and Sen 2006) reported two strains of VRSA and six strains of VISA in the Northen part of India.

Biofilm production by $S$. aureus was found to be $47.8 \%$ strong, $8.955 \%$ moderate and $43.3 \%$ biofilm non producers. Whereas $66.7 \%$ and $33.3 \%$ of isolates were found to be strong and biofilm non-producer from nasal swab specimens respectively. A study, conducted by Mirani et al. (Mirami et al. 2013) reported 57\% of MRSA isolates as biofilm producer. Rewatkar and Wadher (Rewatkar and Wadher, 2013) reported 90\% of strong biofilm producer and remaining $10 \%$ of weak/ none producer.

\section{CONCLUSION}

Higher rate of MRSA was found in female in age group 21-30 years. Amikacin was found to be most effective drug, whereas penicillin $G$ was found as the least effective drug. Upon performing MIC test of MRSA isolates from clinical specimens, 3.5\% (2) of MRSA isolates were found as VRSA and $54.4 \%$ (31) and $42.1 \%$ (24) were as VISA and VSSA respectively. Whereas all nasal swab isolates were sensitive to Vancomycin. From this study, it could be concluded that all of the MRSA isolates i.e. 100\% were multi drug resistant (MDR), which is the significant public health problem in context of Nepal, indicating the high risk of staphylococcal infections in our context. This high load of MDR organisms provokes the necessity of strictly performing susceptibility testing before starting antibiotic therapy, or there may be chance of clinical failure. Thus determination of MIC of Vancomycin is crucial. Inducible Clindamycin resistance test cannot be observed in routinely done antibiotic susceptibility testing by Kirby Bauer method hence specific D-test should be performed before treatment with Clindamycin. There are various methods for detection of biofilm production and both tube test and agar plate methods can be carried out for comparative study.

\section{ACKNOWLEDGEMENTS}

The authors express sincere gratitude to Nabaraj Adhikari, Lecturer, Central Department of Microbiology, Tribhuvan University, Kirtipur, Kathmandu and all the staff members of Manmohan Memorial College and Teaching Hospital, Kathmandu, Nepal and Kantipur College of Medical Science, Sitapaila, Kathmandu.

\section{REFERENCES}

Adaleti R, Nakipoglu Y, Ceran N, tasdemir C, kaya F and Tasdemir S (2010) Prevalence of phenotypic resistance of Staphylococcus aureus isolates to Macrolide, Lincosamide, Streptogramins B, Ketolid and Linezolid antibiotics in Turkey. Braz 


\section{J Infect Dis 14: 11-1.}

Andrews JM (2001) Determination of minimum inhibitory concentration. J Antimicrob Chemother 48: S15-16.

Arch G, Mainous III AG, Hueston WJ and Everett CJ (2006) Nasal carriage of Staphylococcus aureus and methicillin resistance $S$. aureus in the United States. Ann Family Med 4(1): 132-137.

Assadullah S, Kakru DK, Thoker MA, Bhat FA, Hussain $\mathrm{N}$ and Shah A (2003) Emergence of low level vancomycin resistance in MRSA. Indian J Med Microbial 21(3): 196-198.

Bhomi U, Rijal KR, Neupane B, Shrestha S, Chaudhary M, Acharya D, Thapa Shrestha U, Adhikari N and Ghimire P (2016) Status of inducible clindamycin resistance among macrolide resistant Staphylococcus aureus. Afr J Microbiol Res 10(9): 280-284.

Boucher HW and Corey GR (2008) Epidemiology of methicillin-resistant Staphylococcus aureus. Clin Infect Dis 46: 344-349.

Boyce JM, Cookson B, Christiansen K et al. (2005) Methicillin resistance Staphylococcus aureus. Lancet Infect Dis 5: 653-63.

Centers for Disease Control and Prevention (CDC) (2013) Antibiotic resistance threats in the United States 85-86.

Cesur S and Cokca F (2004) Nasal carriage of methicillinresistant Staphylococcus aureus among hospital staff and outpatients. Infect Control Hosp Epidemiol 25: 169-171.

Clinical and Laboratory Standards Institute. Performance Standards for antimicrobial susceptibility testing (2013) twenty-second Informational supplement, CLSI Document M100-S20. Wayne, PA 33.

Cohen PR (2007) Community- acquired methicillinresistant Staphylococcus aureus skin infections; a review of epidemiology, clinical features, management, and prevention. Intel J Dermatol 46: $1-11$.

Donlan RM and Costerton JW (2002) Biofilms: survival mechanism of clinically relevant microorganisms. Clin Microbiol Rev 15: 167-193.
Duerenberg RH, Vink C, Kalenic S et al. (2007) The molecular evolution of Methicillin-Resistant Staphylococcus aureus. Clin Microbial Infect 13: 222-35.

Fiebelkorn KR, Crawford SA, McElmeet ML and Jorgensen JH (2003) Pracrical disk diffusion method for detection of inducible clindamycin resistance in Staphylococcus aureus and coagulasenegative staphylococci. J Clin Microbiol 41: 47404744 .

Foster TJ (2004) The Staphylococcus aureus "superbug". J Clin Invest 114: 1693-6.

Freeman J, Falkiner FR and Keane CT (1989) New method for detecting slime production by coagulase negative staphylococci. J Clin Pathol 42: 872-874.

Gonsu KH, Kouemo SL, Toukam M, Ndze VN and Koulla SS (2013) Nasal carriage of Methicillin resistance Staphylococcus aureus and its antibiotic susceptibility pattern in adult hospitalized patients and medical staff in some hospitals in Cameroon. J Micro Antimicrob 5(3): 29-33.

Kumari N, Mohaprata TM and Singh I (2008) Prevalence of methicillin-resistance Staphylococcus aureus (MRSA) in a tertiary-care hospital in eastern Nepal. J Nepal Med Assoc 47(170): 53-6.

Lai CF, Liao CH, Pai MF, Chu FY, Hsu SP, Chen HY, Yang JY, Chiu YL, Peng YS, Chang SC, Hung KY, Tsai TJ and Wu KD (2011) Nasal carriage of methicillin-resistant Staphylococcus aureus is associated with higher all - cause mortality in haemodialysis patients. Clin J Am SocNephrol 6: 167-174.

Lowy FD (1998) Staphylococcus aureus infections. New Engl J Med 339: 520-32.

Lu PL, Tsai JC, Chiu YW, Hsiao CF and Siu LK (2008) Methicillin-resistant Staphylococcus aureus carriage, infection and transmission in dialysis patients, healthcare workers and their family members. Nephrol Dial Transplant 23: 1659-1665.

Lucet JC, Chevret S, Durand-Zaleski I, Chastang C and Regnier B (2003) Prevalence and risk factors for carriage of methicillin resistant Staphylococcus aureus at admission to the intensive care unit. Arch Intern Med 163: 181-188. 
Magiorakos AP, Srinivasan A, Carey RB, Carmeli Y, Falagas ME, Giske CG,Harbarth S, Hindler JF, Kahlmeter G, Olsson-Liljequist B, Paterson DL, Rice LB, Stelling J, Struelens MJ, Vatopoulos A, Weber JT and Monnet DL (2012) Multidrugresistant, extensively drug-resistant and pandrugresistantbacteria: an international expert proposal for interim standard definitions for acquired resistance. Clin Microbiol Infect 18: 268-281.

Mirani AZ, Aziz M, Khan MN, Lal I, Hassan NU and Khan SI (2013) Biofilm formation and dispersal of Staphylococcus aureus under the influence of oxacillin. Microb Pathog_61-62: 66-72.

Mishra SK (2008) Microbiology of lower respiratory tract infection with special reference to extended beta lactamase and Metallo beta lactamase producing strains among the patients attending at Tribhuwan University Teaching Hospital. Master Thesis, Institute of Medicine, Tribhuwan University, Nepal Med J 127: 78-84.

Pandey S, Raza MS and Bhatta CP (2012) Prevalence and antibiotic sensitivity pattern of methicillinresistant-Staphylococcus aureus in Kathmandu Medical College-Teaching Hospital. Journal of Institute of Medicine 34(1): 13-17.

Pant J and Rai SK (2007) Occurrence of Staphylococcus aureus in hospital environment and staffs in Teaching Hospital in Kathmandu, Nepal. J Nepal Assoc Med Lab Sci 8: 72-73.

Rahimi F, Bourazi M, Katouli M and Pourshafie MR (2013) Antibiotic resistance pattern of methicillin resistant and methicillin sensitive Staphylococcus aureus isolates in Tehran, Iran. Jhundishapur J Microbiol 6(2): 144-149.

Rajbhandari R, Manandhar SP and Shrestha J (2003) Comparative study of MRSA and its antibiotic susceptibility pattern in indoor and outdoor patients of Bir Hospital. Nepalase J Microbiol 1: 6265.

Rewatkar AR and Wadher BJ (2013) Staphylococcus aureus and Pseudomonas aeruginosa-Biofilm formation methods. P.G.T Department of Microbiology, L.I.T Premises, Rasthrasant Tukdoji Maharaj Nagpur University, Nagpur 440033, MaharastraIndia 36-40.
Rijal KR, Pahari N, Shrestha BK, Nepal AK, Paudel B, Mahato P and Skalko-Basnet N (2008) Prevalence of methicillin-resistant Staphylococcus aureus in school children of Pokhara. Nep Med Coll J 10(3): 192-195.

Salah IK, Nagla AA and Ahmed IF (2012) Prevalence and antimicrobial susceptibility pattern of methicillin resistance Staphylococcus in Sudanese surgical ward. Pharmacology and Pharmacy 3(1): 103-108.

Schmid H, Romanos A, Schiffl H and Lederer SR (2013) Persistent nasal methicillin-resistant Staphylococcus aureus carriage in haemodialysis outpatients: a predictor of worse outcome. BMC Nephrol 4: 93

Shah B, Singh AK, Ghosh A and Bal M (2008) Identification and characterization of a vancomycin-resistant Staphylococcus aureus isolated from Kolkata (South Asia). J Med Microbiol 57: 72-79.

Shakya B, Shrestha S and Mitra T (2010) Nasal carriage rate of methicillin-resistant Staphylococcus aureus among at National Medical College Teaching Hospital, Birgunj, Nepal. Nepal Med Coll J 12(1): 26-29.

Sharma P and Vishwanath G (2012) Study of vancomycin susceptibility in methicillin resistant Staphylococcus aureus isolated from clinical samples. Ann Trop Med Public Health 5(3): 178180.

Shrestha B, Pokhrel BM and Mohapatra TM (2009) Antimicrobial susceptibility pattern of nosocomial isolates of Staphylococcus aureus in a tertiary care hospital, Nepal. J Nepal Med Assoc 48(147): 234-8.

Skov R, Smyth R, Larsen AR, Bolmostrom A andKarlsson A (2006) Phenotypic detection of methicillin resistance in Staphylococcus aureus by disk diffusion testing and $\mathrm{E}$ test on Muller-Hinton agar. J Clin Microbiol 44: 4395-9.

Steinberg JP, Clark CC and Hackman BO (1996) Nosocomial and community acquired Staphylococcus aureus bacretemias from 1980 to 1993: impact of intravascular devices and methicillin resistance. Clin Infect Dis 23: 255-9.

Steward CD, Raney PM, Morrell AK, Williams PP, 
Mc Dougal LK and Jevitt L (2005) Testing for induction of Clindamycin resistance in Erythromycin resistant isolates of Staphylococcus aureus. J Clin Microbiol 43: 1716-21.

Tiwari HK and Sen MR (2006) Emergence of Vancomycin resistant Staphylococcus aureus (VRSA) from tertiary care hospital from northen part of India. BMC infect Dis 6: 156-160.

Tiwari HK, DAS AK, Sapkota D, Sivarajan K and Pahwa VK (2009) Methicillin-Resistance Staphylococcus aureus: Prevalence and Antibiogram in a tertiary hospital in Western Nepal. J Infect Dev Ctries 3(9): 681-684.

Vandecasteele SJ, Boelaert JR and De Vries AS (2008) Staphylococcus aureus infections in hemodialysis: What a nephrologist should know. Clin J Am Soc
Nephrol 4: 1388-1440.

Venubabu T, Channappa T, Shivannavar $\mathrm{T}$ and Subhaschandra M (2011) Vancomycin resistance among Methicillin resistant Staphylococcus aureus isolates from intensive care units of tertiary care Hospitals in Hyderabad. Indian J Med Res 134(5): 704-708.

Walsh TR and Howe RA (2002). The prevalence and mechanisms of Vancomycin resistance in Staphylococcus aureus. Annu Reb Microbiol 56: 657675.

Woods CR (2009) Macrolide inducible resistance to Clindamycin and the D-Test. Pediatr Infect Dis J.; 28: 1115-1118. 